\title{
PROKLA-Redaktion
}

\section{Editorial: StadTRaum}

Jahrhundertelang galt die Stadt als Inbegriff von wirtschaftlicher, politischer und vor allem kultureller Entwicklung; gesellschaftliche Dynamik war etwas, das sich vorwiegend in Städten abspielte, auch wenn die dabei ablaufenden Prozesse unterschiedlich beurteilt wurden. Bereits die biblischen bis in die Gegenwart transportierten Bilder von »Babylon《 und »Jerusalem《 verweisen auf diese ambivalenten Einschätzungen: Stadt als Ausdruck von moralischem Niedergang und Dekadenz oder als Verwirklichung von Kultur und Aufklärung. Mit der industriekapitalistischen Stadt des 19. Jhs. erhielt diese Dichotomie eine neue Evidenz: beinhaltete die Stadt doch auf der einen Seite technische Wunderwerke und das Versprechen von Wohlstand, Bildung und die Möglichkeit individueller Entfaltung, auf der anderen Seite aber auch das Elend des Proletariats, die Auflösung familiärer Bindungen und die Entfremdung von den natürlichen Lebensgrundlagen. Wurde das Leben in den Städten auch noch so unterschiedlich beurteilt, so war man sich doch einig in seiner zentralen Rolle für die Entwicklung der Gesellschaft.

Allerdings scheint sich jetzt auch dieser Konsens aufzulösen. So proklamierte jüngst Alain Touraine das Ende der Städte. Er behauptete, daß wir nur noch »glauben, in Städten zu leben... Doch die Stadt, die einst um die wichtigen Handlungsorte - den Palast, den Markt, die Kathedrale - wuchs, ist längst Vergangenheit« (Das Ende der Städte? Die Zeit Nr. 23, 24.5.1996). Vor allem traut er dem Städtischen nicht mehr die wichtigen sozialen Integrationsleistungen zu, die die Grundlage weiterer Entwicklung darstellen. Damit nimmt Touraine die weltweit sichtbaren Trends der inneren Auflösung des städtischen Zusammenhangs auf, von dem die Flucht der Mittel- und Oberschichten in die Vorstädte nur der deutlichste Ausdruck ist. Städte scheinen am Ende des 20. Jhs. zu bloßen Standorten zu verkommen. Die Kehrseite ihrer Einbeziehung in die sich verdichtenden globalen Prozesse ist anscheinend ihr Ende als gesellschaftliche Veranstaltung.

Die Entwicklung vor allem in den nordamerikanischen Mega-Städten (die, wie oft angenommen wird, die Zukunft europäischer Städte vorwegnehmen) schien solche Vorstellungen bis vor kurzem noch eindeutig zu bestätigen. Inzwischen zeichnen sich aber auch andere Entwicklungslinien ab. Roger Keil argumentierte auf dem letzten Deutschen Politologentag im Oktober 1997, daß gerade die städtische Gesellschaft aufgrund ihrer neuen Vielfalt und Dynamik zu einem wichtigen Integrationsmechanismus des glo- 
balen Zeitalters geworden sei. Auf den Analysen von Saskia Sassen' aufbauend betonte er nicht nur eine neue Qualität von Urbanität, sondern auch die auf lokaler Ebene entstehenden neuen Formen des Managements sozialer Konflikte, die gleichzeitig die globale Konkurrenzfähigkeit steigern sollen.

Dieses neue Zusammenspiel von globalen und lokalen Faktoren wird die frühere Bedeutung des Städtischen nicht einfach wiederherstellen, neue Erklärungsansätze sind erforderlich. Mit dieser Nummer versucht die PROKLA den Spannungsbogen zwischen der Stadt als bloß räumlicher Konzentration (»StadtRaum《) einerseits und als Ort neuer Bedeutungen und neuer Handlungsmöglichkeiten andererseits (»StadTraum《) auszuloten. Wir widmen uns also der Stadt nicht nur, weil der Prozeß zunehmender Verstädterung unaufhaltsam voranschreitet. In der letzten PROKLA (Landwirtschaft) wurde bereits daran erinnert, daß im Jahr 1800 knapp 2,5\%, im Jahr 1900 bereits 10\% der Menschheit weltweit in Städten lebten, daß es aber im Jahr 2000 mehr als $50 \%$ sein werden. Vor allem die westlichen Gesellschaften sind vornehmlich städtische Gesellschaften geworden. Gerade die jüngsten Entwicklungen, die der Stadt eine neue Bedeutung zuweisen, verdanken sich aber nicht diesen quantitativen Bewegungen. Die im Zuge der Globalisierungsprozesse stattfindende »Erosion des Nationalstaats $\ll$ ist kein eindimensionaler, linearer Prozeß (was in einem allgemeinen Kontext auch schon das Thema von Michael Mann in PROKLA 106 Konflikte um den Wohlfahrtsstaat war). Für die Städte ist dabei vor allem die Ten-

1 Losing Control? Sovereignty in an Age of Globalization. New York, 1996. denz zur »Glokalisierung《 relevant, der Verlagerung von immer mehr Entscheidungsoptionen auf subnationale Ebenen, womit allerdings eine Reihe massiver Folgeprozesse und Folgeprobleme verbunden sind. Vor allem haben die Ausdifferenzierung und die Unterschiede zwischen städtischen Regionen enorm zugenommen, Städtesysteme haben neue Hierarchien ausgebildet; Veränderungen in den politischen Regimen werden konstatiert; eine neue Zusammenballung sozialer Probleme und Polarisierungen sowie neue Umweltprobleme werden in den Städten ausgemacht.

Wenige dieser Trends waren vor zehn Jahren - als PROKLA 68 (Lokaler Staat und Kommunalpolitik) erschien bereits Thema. Aber wie damals geht es der PROKLA vor allem um die Rolle politischer Prozesse und um die Folgen von Fordismuskrise und aktuellen globalen Restrukturierungen für die lokale Politik - was im Gegensatz zu Ökonomie und Sozialstruktur gleichzeitig die in der Global-City-Literatur am wenigsten beleuchtete Ebene der heutigen Stadtentwicklung ist.

Schien die Bedeutung transnationaler ökonomischer Prozesse für die Entwicklung lokaler Politik vor zehn Jahren noch wenig relevant, so interessiert uns heute die Art und Weise ihrer Interaktion mit den nationalen und regionalen »governance«-Systemen, weil klar geworden ist, daß auch globale Prozesse sich letztlich an einem Ort materialisieren. Selbst wo »rein « lokale Prozesse untersucht werden, ist deshalb zunehmend eine transnationale Untersuchungsperspektive einzunehmen.

Neil Brenner veranschaulicht an Hand der Entwicklung der Raumordnungspolitik der BRD, daß die globale Dimension der Globalisierungsprozesse schlechterdings nicht von den sub- 
globalen geographischen Ebenen gelöst werden kann, auf welchen und durch welche sie sich territorialisiert. So kontrastiert er die 60er Jahre, in denen die internen Peripherien und ländlichen Regionen im Vordergnund der zentralstaatlichen Raumplanung standen und es um eine möglichst gleichmäßige Verteilung des Surplus über das nationale Territorium ging, mit der in den 70er Jahren beginnenden Phase, in der staatliche Planungspolitik sich zunehmend auf endogene, städtisch-regionale Wachstumspotentiale bezieht. Dieser Planungspolitik entsprechend begannen sich »unternehmerische« Formen 10kaler und regionaler politischer Regime auszubilden, die sich auf die Förderung der städtisch-regionalen Entwicklung konzentrieren und weder Bezug auf das ländliche Hinterland nehmen noch auf die makroökonomischen Steuerungsversuche des Zentralstaats. Damit einher geht auch eine Wandlung in der Stadtplanung weg von der Flächensanierung hin zu kleinteiligen und flexiblen Strategien, die zunehmend von parastaatlichen Institutionen organisiert und ausgeführt werden. Zusammengenommen ergeben diese verschiedenen Tendenzen eine Neudimensionierung der Räume, auf die sich politische Prozesse und Konflikte beziehen.

Die Materialisierung globaler Prozesse an einem Ort produziert, so Saskia Sassen im Oktober auf dem Politologentag, häufig eine regulatorische Leerstelle, welche die Städte (und die in ihnen agierenden globalen Märkte und Firmen) zu füllen versuchen. In den dabei entstehenden städtischen Regulationsmodi bilden sich möglicherweise tragende Elemente eines neuen Akkumulationsregimes heraus, wobei neofordistische von postfordistischen $\mathrm{Va}$ rianten unterschieden werden müssen. Die erste Variante wäre eine Verschär- fung fordistischer Regulation, die auf einer Re-Metropolisierung der städtischen Formen (in einer desorganisierten Gesellschaft) beruht, während die postfordistische Variante, so Alain Lipietz, auf einer yorganisierten Mobilisierung des Territoriums « beruht und eine Kooperationsform beinhaltet, wdie auf Verhandlungen, auf Verhältnissen von Angesicht zu Angesicht, also wieder auf Nähe basiert. ${ }^{2}$

In einer »Anwendung « der an avancierten "global cities" gewonnenen Analysen postfordistischer Stadtentwicklung auf das neue, wiedervereinigte Berlin zeigt Margit Mayer, inwiefern auch diese definitive $»$ NichtWeltstadt« von den Trends zu Internationalisierung, De- und Reindustrialisierung, räumlicher Rekonfiguration und neuartiger sozialer Polarisierung beeinflußt wird. Berlins Partnerstadt Los Angeles eignet sich als Kontrast und Vergleichsmodell, weil - wie Paul Krugmann feststellt - »...these days every place looks a lot like L.A. ¿ $^{3}$

Ein wesentliches Merkmal der neuen Stadtpolitik ist der Umbau des lokalen Wohlfahrtsstaats, der mit einer Erosion traditioneller sozialer Rechte gekoppelt ist. Die Konsequenz davon ist, daß die sozialen Polarisierungsprozesse, die durch den globalen »race to the bottom « ausgelöst werden, zwar national und regional recht unterschiedlich verlaufen, aber immer weniger durch sozialstaatliche Maßnahmen kompensiert werden. Das zeigt nicht nur der Fall Berlin (dem wir im nächsten Jahr ein ganzes Heft widmen werden), sondern wird auch durch die deutsche Segregations-

2 Zur Zukunft der städtischen Ökologie, in: M. Wenz (Hg.), Stadt-Räume. Fft. Campus 1991, S. 134.

3 Pop Internationalism, Cambridge, Mass. MIT: 1996, S. 210. 
forschung dokumentiert. Hierzu liefert Jens Dangschat einen Überblick zum »state of the art«. Dabei zeigt sich, daß die gewandelte Rolle des Raumes auch in die Kategorien der Forschungsansätze selbst gewandert ist: Ging es der Theorie residentieller Segregation zunächst um die Übertragung sozialer Ungleichheit in den Raum, wobei dieser lediglich Indikator für soziale Segmentierung war, so wird er heute als entscheidender Motor für die Reproduktion sozialer Ungleichheit gesehen.

Bislang in der Stadtforschung weitgehend unterbelichtet sind die Auswirkungen von Globalisierung und Fordismuskrise auf die natürliche und die erbaute Umwelt der Stadt. Dabei beobachten John Graham und Roger Keil, daß städtische Entwicklung nicht mehr gegen, sondern zunehmend mit Hilfe von »Natur《 stattfindet. Während erstmals Natur global dem Kapitalverhältnis unterworfen wird, entdecken Planer und Städtebauer Natur als Medium der Konstruktion von Urbanität, werden lokale klimatische, topographische und ökologische Bedingungen zunehmend zu Voraussetzungen städtischer Planung und städtischen Designs gemacht. Graham und Keil illustrieren mit Beispielen aus der Region Toronto, daß sich die fordistische Form der Integration von Stadt und Natur in der suburb aufgelöst hat zugunsten einer neuen Form der Subordinierung von Natur, in der »wilde《 und »natürliche« Orte in die Urbanität importiert werden und wo die Stadtplanung sich gezwungen sieht, ein gewisses Umweltmanagement in der Praxis zu berücksichtigen - allerdings ohne das Nachhaltigkeitsproblem der fordistischen Periode zu lösen.

Der Globalisierungsdiskurs insinuiert, daß die genannten Umstrukturierungen und Veränderungen weltweit gelten.
Aber sowohl für die Städte des Südens als auch für die Städte in postsozialistischen Ländern gilt zwar, daß auch sie sich nun auf neue Arten in die weltweite Konkurrenz integriert finden. Aber die Dynamik ihrer Entwicklung, vor allem ihrer politischen Entwicklung, hängt noch immer stark von je unterschiedlichen, historisch kontingenten, Kräften ab. Andererseits können auch überraschende Ähnlichkeiten festgestellt werden wie Michael Brie in seiner Untersuchung des urbanen Regimes, das sich im Moskau der 90er Jahre herausgebildet hat, demonstriert: klientelistische Verquickungen und die ihnen entsprechenden politischen und ökonomischen Strukturen lassen sich hier mit dem Konzept der »urban machine« analysieren, welches ursprünglich zum Verständnis US-amerikanischer Städte des ausgehenden 19. Jhs. entwickelt wurde.

Außerhalb des Schwerpunkts erscheint von Lutz Hieber ein Beitrag über die Protest- und Aufklärungsformen USamerikanischer Aids-Aktivisten. Im Zentrum dieses Beitrages steht die Verbindung von Politik und Kunst, wie sie sich vor allem in New York um die Gruppe ACT-UP herausgebildet hat. Auch dies ist eine Facette unter bestimmten historischen Bedingungen entstandener, städtischer Kultur.

$* * *$

Zu den Autoren des vor zehn Jahren erschienen Stadtheftes (PROKLA 68 Lokaler Staat und Kommunalpolitik) gehörte auch Karl Homuth. Dieser engagierte Soziologe und Stadtforscher, der stets darum bemüht war, Wissenschaft und praktisches politisches Handeln miteinander zu verbinden, ist am 6. Oktober im Alter von 48 Jahren verstorben. Seinem Andenken widmen wir dieses Heft. 